\section{Elterliche Alkoholbelastung und die Entwicklung von Suchtproblemen bei ihren Kindern - Ergebnisse der prospektiv-longitudinalen EDSP-Studie ${ }^{1}$}

Zusammenfassung: Anliegen: Es werden Assoziationen zwischen elterlicher Alkoholbelastung und der Häufigkeit und des Erstmanifestationsalters des Konsums, des Missbrauchs sowie der Abhängigkeit von legalen und illegalen Drogen bei Jugendlichen berichtet. Methode: Die Ergebnisse basieren auf Daten der Early Developmental Stages of Psychopathology-(EDSP-)Studie, einer epidemiologischen Längsschnittstudie, in welcher eine repräsentative Stichprobe von Jugendlichen und jungen Erwachsenen prospektiv u.a. hinsichtlich der Entwicklung von Substanzkonsum und Substanzstörungen untersucht wurde. Der EDSP angeschlossen war eine direkte Elternbefragung. Als Diagnoseinstrument wurde ein standardisiertes Interview, das M-CIDI, verwendet. Die Befunde basieren auf einer Stichprobe von 917 Jugendlichen, für welche 4-Jahres-Follow-up-Daten vorliegen und deren Eltern an der Elternbefragung teilnahmen. Ergebnisse: Elterliche Alkoholbelastung ist mit höherem Alkoholkonsum, häufigerem regelmäßigen Rauchen sowie häufigerem Konsum illegaler Drogen bei Jugendlichen assoziiert. Es finden sich ebenfalls Assoziationen zu Missbrauch und Abhängigkeit von legalen und illegalen Drogen, wie auch speziell zum Übergang vom gelegentlichen in den regelmäßigen Alkoholkonsum. Das Einstiegsalter in den schädlichen Alkoholkonsum und in regelmäßiges Rauchen liegt bei Jugendlichen mit zwei betroffenen Eltern merklich früher als bei Kindern ohne belastete Eltern. Schlussfolgerungen: Elterliche Alkoholbelastung stellt einen potenten Risikofaktor für die frühe Entwicklung von Suchtproblemen dar. Bei Kindern alkoholbelasteter Eltern sollte mittels spezifischer Präventions- und Interventionsmaßnahmen bereits in frühen Stadien der Suchtentwicklung vorgebeugt werden.

Schlüsselwörter: Epidemiologie - Konsum, Missbrauch, Abhängigkeit von legalen und illegalen Drogen - Elterliche Alkoholbelastung - Risikofaktoren

Parental Alcoholism and the Risk of Substance Use, Abuse and Dependence in Offspring: Findings from the Prospective-longitudinal EDSP Study: Objective: To examine the associations between parental alcohol use disorders and patterns of substance use and DSM-IV substance use disorders in offspring. Methods: Data come from the prospective longitudinal Early Developmental Stages of Psychopathology

Suchttherapie 2001; 2: 125-136

(c) Georg Thieme Verlag Stuttgart · New York ISSN 1439-9903
Roselind Lieb ${ }^{1}$, Barbara Isensee ${ }^{1}$, Michael Höfler ${ }^{1}$, Hildegard Pfister ${ }^{1}$, Hans-Ulrich Wittchen ${ }^{1,2}$

${ }^{1}$ Max-Planck-Institut für Psychiatrie, Arbeitsgruppe Klinische Psychologie und Epidemiologie, München

2 Technische Universität Dresden, Klinische Psychologie und Psychotherapie, Dresden
(EDSP) Study of a representative community sample of adolescents and young adults. Use, DSM-IV abuse and dependence of legal and illegal drugs were assessed by using a standardized interview (M-CIDI). In a separate family supplement, direct interviews were conducted with the parents. Data are based on 917 subjects who completed the whole study period and for whom direct diagnostic information from the parents could be assessed. Results: Parental alcohol use disorders could be shown as predictors for increasing levels of alcohol consumption, for regular smoking and higher use of illegal drugs in offspring. They further predicted progression from occasional into regular use of alcohol and were associated with abuse and dependence of legal and illegal drugs. For harmful use of alcohol and for regular smoking, age of onset was earlier in offspring with both parents affected. Conclusions: Parental alcoholism predicts escalation of substance use and the development of substance use disorders in the offspring. The offspring should be considered as a special high risk group for which specific prevention and treatment programs should be offered.

Key words: Epidemiology - Use, Abuse, Dependence of Alcohol, Nicotine and Illicit Drugs - Parental Alcohol Use Disorders - Risk Factors

\footnotetext{
${ }^{1}$ Diese Arbeit ist im Rahmen der Early Developmental Stages of Psychopathology-(EDSP-)Studie entstanden, welche im Rahmen des Forschungsschwerpunktes „Biologische und psychosoziale Faktoren von Drogenmissbrauch und Drogenabhängigkeit" des Bundesministeriums für Bildung, Forschung und Technologie (BMBF, 01 EB 9405/6 und 01 EB 9901/6) gefördert wurde. Die Projektleiter sind Prof. Dr. Hans-Ulrich Wittchen und Dr. Roselind Lieb. Wissenschaftliche Projektmitarbeiter/-innen sind oder waren: Dr. Kirsten von Sydow, Dr. Gabriele Lachner, Dr. Axel Perkonigg, Dr. Peter Schuster, Dr. Franz Gander, Dipl.-Stat. Michael Höfler und Dipl.-Psych. Holger Sonntag. Als weitere wissenschaftliche Mitarbeiter/-innen sind oder waren beteiligt: Mag. phil. Esther Beloch, Mag. rer. nat. Martina Fuetsch, Dipl.-Psych. Elzbieta Garczynski, Dipl.-Psych. Alexandra Holly, Dipl.-Psych. Barbara Isensee, Dr. Marianne Mastaler, Dr. Chris Nelson, Dipl.-Inf. Hildegard Pfister, Dr. Victoria Reed, Dipl.-Psych. Dilek Türk, Dipl.-Psych. Antonia Vossen, Dr. Ursula Wunderlich und Dipl.-Psych. Petra Zimmermann. Wissenschaftlich beraten wurde die EDSP u.a. von Prof. Dr. Jules Angst (Zürich), Prof. Dr. Jürgen Margraf (Basel), Prof. Dr. Günther Esser (Potsdam), Prof. Dr. Kathleen Merikangas (Yale, New Haven) und Prof. Dr. Ron Kessler (Harvard, Boston).
} 


\section{Einleitung}

Der Konsum von Alkohol und alkoholbedingte Störungen gehören in den westlichen Ländern zu den häufigsten Gesundheitsproblemen im Erwachsenen- wie auch im Jugendalter $[1,2]$. In den letzten 20 Jahren wurde deshalb die Erforschung von Faktoren, welche die Entwicklung von Alkoholproblemen erklären können, verstärkt zum Gegenstand wissenschaftlichen Interesses (Reviews siehe [3, 4]). Im Blickfeld einer Reihe von Studien stand hierbei die Frage, inwiefern familiäre bzw. elterliche Alkoholprobleme als Risikofaktor für die spätere Entwicklung von Suchtproblemen bei ihren Kindern zu sehen sind. Inzwischen liegt zu dieser Frage eine Reihe von Befunden vor, welche relativ eindeutig darauf hinweisen, dass Personen mit einer elterlichen Alkoholbelastung im Vergleich zu Personen ohne eine solche Belastung einem beträchtlich höheren Risiko ausgesetzt sind, ebenfalls Alkoholprobleme $\mathrm{zu}$ entwickeln [5-17]. Ebenso legt eine Reihe von Befunden nahe, dass Kinder von Eltern mit einer Alkoholbelastung nicht nur anfälliger für die Entwicklung von Alkoholproblemen sind, sondern ebenfalls auch ein erhöhtes Risiko zeigen, Probleme im Umgang mit anderen psychotropen Substanzen zu entwickeln $[9-11,18,19]$.

Obwohl durchaus Befunde aus anspruchsvollen Längs- und High-Risk-Studien zur Frage familiärer Assoziationsmuster zwischen elterlicher Alkoholbelastung und der Entwicklung von Substanzproblemen bei ihren Kindern vorliegen [8, 12, 18], besteht ein Hauptkritikpunkt an der bisherigen Forschung darin, dass - mit einigen wenigen Ausnahmen, wie etwa den Arbeiten von Sieber und Angst [5], Russell et al. [6], Mathew et al. [9] und Kendler et al. [13] - solche Assoziationsmuster fast ausschließlich an klinischen Stichproben, d.h. über die Untersuchung von Betroffenen aus Behandlungseinrichtungen, ermittelt wurden. Klinische Studien haben jedoch den Nachteil, dass deren Ergebnisse aufgrund einer Stichprobenselektivität verzerrt sein können und deshalb möglicherweise zu falschpositiven Befunden führen, somit zu einer Überschätzung der familiären Assoziationen [20]. Die Verallgemeinerung auf Personen außerhalb klinischer Einrichtungen ist daher nicht unbedingt gewährleistet.

Im vorliegenden Beitrag werden ausgewählte Ergebnisse zu familiären Assoziationen von Suchtproblemen aus der Early Developmental Stages of Psychopathology-Studie berichtet, in welcher eine repräsentative Stichprobe 14- bis 24-Jähriger prospektiv in drei Untersuchungswellen befragt wurde und eine zusätzliche Befragung der Eltern erfolgte. Fokussierend auf die jüngere Kohorte, die 14- bis 17-Jährigen, werden in diesem Beitrag nach der Darstellung von Basis- und 4-JahresVerlaufsergebnissen für Substanzkonsum sowie substanzbedingtem Missbrauch und Abhängigkeit folgende Fragen beantwortet:

1. Welche Zusammenhänge zeigen sich zwischen elterlicher Alkoholbelastung und dem Konsum von legalen und illegalen Drogen bei den Kindern?

2. Welche Zusammenhänge bestehen zwischen elterlicher Alkoholbelastung und dem Vorliegen von DSM-IV-Missbrauch und Abhängigkeit von legalen und illegalen Drogen bei den Kindern?

3. Ist elterliche Alkoholbelastung speziell mit dem Übergang in bestimmte Stadien des Konsums von Alkohol bei den Kindern assoziiert?
4. Zeigen sich Assoziationen zwischen elterlicher Alkoholbelastung und dem Alter bei erstmaligem schädlichen Alkoholkonsum, erstmaligem regelmäßigen Rauchen sowie erstmaligem Konsum illegaler Drogen? Zeigen sich Assoziationen zwischen elterlicher Alkoholbelastung und dem erstmaligen Auftreten von substanzbedingtem Missbrauch und Abhängigkeit?

\section{Methoden}

\section{Studie und Design}

Die Befunde wurden im Rahmen der Early Developmental Stages of Psychopathology-(EDSP-)Studie ermittelt, deren Ziel es ist, auf der Grundlage einer repräsentativen Stichprobe von Jugendlichen und jungen Erwachsenen zu untersuchen, a) wie häufig und unter welchen Umständen diese psychotrope Substanzen gebrauchen, missbrauchen oder eine Abhängigkeit entwickeln, b) welche Muster von Stadienwechseln zwischen Gebrauch, Missbrauch und Abhängigkeit zu beobachten sind und c) welche Vulnerabilitäts- und Protektionsfaktoren in solchen Frühstadien für die Entwicklung von Drogenkonsum, Missbrauch und Abhängigkeit relevant sind. Detaillierte Beschreibungen des methodischen Vorgehens der EDSP finden sich bei Wittchen et al. [21] und Lieb et al. [22].

\section{Stichprobe}

Die EDSP-Studie beruht auf einer Zufallsstichprobe von 14- bis 24-jährigen Jugendlichen und jungen Erwachsenen der Münchener Stadt- und Landkreise, die Ende 1994 aus den Einwohnermelderegistern gezogen wurde. Von den gezogenen 4809 Personen wurden 4263 Personen in die Studie aufgenommen (siehe im Detail hierzu [21, 22]). Bei der 1995 durchgeführten Basisuntersuchung (T0) wurden $\mathrm{n}=3021$ Personen befragt (Ausschöpfungsrate $=71 \%$ ). Von den 1395 14- bis 17-jährigen Jugendlichen der Basisuntersuchung wurden in der ersten Follow-up-Untersuchung (T1; im Mittel 20 Monate nach T0) 1228 Jugendliche ein weiteres Mal untersucht (1996/1997; Ausschöpfungsrate =88\%). Parallel zu dieser ersten Nachuntersuchung wurde eine separate Elternuntersuchung durchgeführt, in der alle Eltern von T1-Teilnehmer(inne)n befragt wurden. Es wurden primär die Mütter untersucht, da neben Informationen zur familiären Psychopathologie Informationen zu Schwangerschaftskomplikationen sowie frühkindlichen Auffälligkeiten der Index-Probanden erfasst werden sollten. 1053 Eltern (1026 Mütter, 27 Väter; Ausschöpfung: 86\%) wurden erfolgreich befragt. In der zweiten Follow-up-Untersuchung (T2, 1998/1999; im Mittel 42 Monate nach T0), welche wieder alle Proband(inn)en der Basisuntersuchung umfasste, wurden 2548 Personen abschließend untersucht (Ausschöpfungsrate $=84 \%$ ).

Bei den im Folgenden angeführten Befunden zu Assoziationen zwischen elterlicher Alkoholbelastung und Substanzgebrauch, -missbrauch und -abhängigkeit bei den Kindern beschränken wir uns auf die zu T0 14- bis 17-jährigen Proband(inn)en, welche an allen drei Untersuchungswellen (T0, T1 und T2) teilnahmen, von welchen ein Elternteil direkt befragt wurde und für die Angaben zu beiden Eltern vorliegen $(\mathrm{n}=917)$. Diese Untergruppe unterscheidet sich bzgl. der Ausschöpfungsraten in den einzelnen Wellen nicht von der Gesamtgruppe [22]. 


\section{Erhebungsinstrumente und diagnostische Einordnung}

\section{Probanden und Probandinnen}

Alle Untersuchungsvariablen wurden mit einer computerisierten Version des Münchener Composite International Diagnostic Interview (M-CIDI) [23], einer modifizierten Version des WHO-CIDI (Version 1.2) [24], erhoben, welches die standardisierte Erfassung von Symptomen, Syndromen, Diagnosen sowie Verlaufsmerkmalen ausgewählter psychischer Störungen nach den Kriterien von ICD-10 [25] und DSM-IV [26] erlaubt. In T0 wurde die Lifetimeversion eingesetzt, welche 12-Monats-Querschnitts- und Lebenszeit-Informationen liefert. In T1 und T2 wurde die 12-Monats-Intervallversion herangezogen, die den 12-Monats-Zeitraum vor der jeweiligen Welle und das restliche Zeitintervall zwischen der vorhergehenden und der aktuellen Untersuchung abbildet. Das M-CIDI beinhaltet ein sog. „Listenheft“, welches Fragebogen zur Erfassung psychologischer Konstrukte enthält. Detaillierte Übersichten hierzu sowie Befunde zur Reliabilität und Validität des M-CIDI liegen bereits vor [21, 22, 27-29].

Auf bereits publizierte Beschreibungen der einzelnen Substanzsektionen (Nikotin, Alkohol, illegale Drogen) des M-CIDI, über welche sowohl der Konsum als auch Missbrauch und Abhängigkeit von Substanzen erfasst werden, soll lediglich verwiesen werden [2,30-33]. Angegeben werden sollen an dieser Stelle (siehe Tab. 1) die Operationalisierungen der für die verschiedenen Substanzen verwendeten Konsumkategorien, welche in diesem und auch in bereits publizierten Beiträgen zur Analyse des Substanzkonsums herangezogen wurden [2, 27, 32-35].

\section{Diagnostische Angaben über die Eltern}

Im Projektteil „Familiengenetik und Sozialisation“ wurde zur direkten diagnostischen Befragung der Eltern eine modifizierte Version des M-CIDI (M-CIDI-FG) [36] herangezogen. Das M-CIDI-FG beinhaltet ein sog. „family-history“-Modul [37], über welches Informationen über Substanzmittelkonsum, Missbrauch und Abhängigkeit sowie weitere psychopatholo- gische Informationen über den nicht befragten Elternteil (in der Regel den Vater) und weitere Familienmitglieder (Großeltern, Geschwister) erhoben wurden (vgl. hierzu [22]). Zusätzlich zu dieser direkten Elternbefragung wurden alle Proband(inn)en zu T0 und T2 über ein solches „family-history“-Modul zu psychopathologischen Auffälligkeiten bei ihren Eltern und Geschwistern befragt. Angaben zur Validität der über dieses Modul erhobenen Informationen über die elterliche Alkoholbelastung finden sich in Lieb et al. [35].

Für die elterliche Alkoholbelastung liegen somit Informationen sowohl von dem befragten Elternteil selbst als auch von den Jugendlichen vor. Zur Auswertung der familiären Assoziationen wurden sowohl die indirekten als auch die direkten diagnostischen Informationen herangezogen [22] und $\mathrm{zu}$ einem Index für elterliche Alkoholbelastung aggregiert. Aufgrund des Überwiegens von indirekten „family-history“-Informationen wurde dabei nicht mehr unterschieden zwischen elterlichem Alkoholmissbrauch und -abhängigkeit, sondern das Berichten von DSM-IV-Missbrauchs- oder Abhängigkeitssymptomen wurde zusammengefasst als „elterliche Alkoholbelastung“ gewertet, wobei die Höhe des elterlichen Alkoholkonsums gemäß den DSM-Vorgaben unberücksichtigt blieb.

\section{Interviewer/-innen, Training und Durchführung der Feldarbeit}

Alle Befragungen wurden von klinisch geschulten Interviewer(inne)n (in der Mehrzahl Diplom-Psycholog(inn)en) durchgeführt. Während der Feldarbeit wurden alle Interviewer/-innen kontinuierlich von klinischen Editorinnen supervidiert, die jedes Interview auf Vollständigkeit, korrekte Durchführung und Kodierung sowie auf sonstige aufgetretene Probleme überprüften. Detaillierte Darstellungen der Feldarbeit finden sich in Wittchen et al. [21] sowie Lieb et al. [22].

\section{Auswertung}

Alle Auswertungen wurden mit gewichteten Daten durchgeführt. Hierbei bekommt jede Beobachtung ein statistisches Gewicht, das auf der inversen Auswahlwahrscheinlichkeit

Tab. 1 Konsumkategorien für die einzelnen untersuchten psychotropen Substanzen

\begin{tabular}{|c|c|c|}
\hline Substanz & Konsumkategorie & Operationalisierung \\
\hline \multirow[t]{4}{*}{ Alkohol } & kein/seltener Konsum & $\begin{array}{l}\text { nie oder nie mehr als } 11 \text {-mal innerhalb von } 12 \text { Monaten Alkohol getrunken } \\
\text { (d.h. nie monatlicher Konsum) }\end{array}$ \\
\hline & gelegentlicher Konsum & $\begin{array}{l}\text { mindestens } 12 \text {-mal pro Jahr, aber nie mehr als dreimal pro Monat } \\
\text { (d.h. mindestens monatlicher, aber nie wöchentlicher Konsum) }\end{array}$ \\
\hline & regelmäßiger Konsum & mindestens viermal pro Monat, aber nie schädlicher Konsum \\
\hline & schädlicher Konsum & $\begin{array}{l}\text { in der Periode des stärksten Konsums Frauen mindestens } 20 \mathrm{~g} \\
\text { und Männer mindestens } 40 \mathrm{~g} \text { Alkohol pro Tag getrunken }\end{array}$ \\
\hline \multirow[t]{2}{*}{ Nikotin } & kein/seltener Konsum & nie geraucht oder nie mindestens 4 Wochen täglich geraucht \\
\hline & regelmäßiger Konsum & mindestens 4 Wochen täglich geraucht \\
\hline \multirow[t]{3}{*}{ illegale Drogen ${ }^{1}$} & kein Kosum & nie eine illegale Droge konsumiert \\
\hline & gelegentlicher Konsum & 1- bis 4-mal im Leben eine illegale Droge konsumiert \\
\hline & regelmäßiger Konsum & mindestens 5-mal im Leben eine illegale Droge konsumiert \\
\hline
\end{tabular}

${ }^{1}$ beinhalten Cannabinoide, Stimulanzien/Amphetamine (Ecstasy, Speed etc.), Opiate, Kokain, Phencyclidine, Psychedelika/Halluzinogene und Inhalantien/ Lösungsmittel 
basiert. Damit wird die Verteilung der Variablen Alter, Geschlecht und Gebiet an die Verteilung in der zugrunde liegenden Population angeglichen. Die Auswertungen wurden mit dem Programmpaket STATA [38] durchgeführt, welches robuste Schätzungen von Standardfehlern und Konfidenzintervallen (KI) bei gewichteten Daten ermöglicht.

Bezüglich der untersuchten Häufigkeiten wird die LifetimePrävalenz zu T0 (prozentualer Anteil der Population der zu T0 14- bis 17-Jährigen, der zu T0 jemals im Verlauf des Lebens das Merkmal gezeigt hat), die Inzidenz T0-T2 (prozentualer Anteil der Population, der zu T0 das jeweils untersuchte Merkmal nicht aufwies, es aber während der Follow-up-Periode erstmalig gezeigt hat) und die kumulierte LebenszeitInzidenz zu T2 (prozentualer Anteil der Population, der zum Zeitpunkt T2 jemals im Verlauf des Lebens das Merkmal gezeigt hat) berichtet.

Für die Assoziationsberechnungen wurden als Grundlage der Einteilung der Probanden in die Konsum- und Störungskategorien die Angaben aus T0, T1 und T2 kumuliert. Für die Analyse der Progression des Alkoholkonsums (Übergang von einer Konsumkategorie in eine höhere) in Abhängigkeit der elterlichen Belastung wurden Untergruppen gebildet, die die Probanden mit höherem Konsum nur unter den Probanden der nächstniedrigeren Konsumkategorie umfassten, z.B. die Gruppe der Probanden mit schädlichem Konsum unter den Probanden mit mindestens regelmäßigem Konsum.

Zur Analyse des Zusammenhangs zwischen elterlicher Alkoholbelastung und dem Outcome bei den Probanden wurden über logistische Regressionen sowie Erweiterungen verschiedene Assoziationsmassen berechnet. Für dichotome Outcome-Maße (z.B. Alkoholabhängigkeit ja/nein) wurden Odds Ratios (OR) ermittelt, für mehrfach gestufte Outcome-Maße (z.B. kein - gelegentlicher - regelmäßiger Konsum illegaler Substanzen) wurden Cumulative Odds Ratios (CUMOR) berechnet [39]. Die Progression in höhere Konsumkategorien wurde über Continuation Odds Ratios (COR) auf ihre Assoziation mit elterlicher Alkoholbelastung geprüft [40]. Alle Assoziationen wurden nach Alter und Geschlecht des Probanden kontrolliert. Mögliche Unterschiede in den Zusammenhängen zwischen Probanden und Probandinnen wurden über Interaktionseffekte mit dem Geschlecht geprüft.

Um zu bestimmen, ob sich die elterliche Alkoholbelastung auf das Alter bei Beginn von Substanzkonsum und -störungen bei den Kindern auswirkt, wurde das Erstmanifestationsalter über Survivalanalysen analysiert. Tests auf Unterschiede zwischen Survivalkurven für Probanden mit und ohne elterliche Alkoholbelastung wurden mit Cox-Regressionen durchgeführt, welche Unterschiede mit Hazard Ratios (HR) darstellen [41]. Um zu prüfen, ob in der Gruppe mit elterlicher Belastung die Erstmanifestation früher erfolgt, wurden Interaktionen Alter $*$ elterliche Alkoholbelastung berechnet.

\section{Ergebnisse}

Prävalenz und Inzidenz von Konsum, Missbrauch und Abhängigkeit legaler und illegaler Drogen bei 14- bis 17-jährigen Jugendlichen

Konsum

Lifetime-Prävalenz zu T0: Zum Zeitpunkt der Basisuntersuchung (1995) berichtet etwa ein Drittel aller 14- bis 17-Jährigen, irgendwann in ihrem Leben gelegentlich Alkohol getrunken zu haben (siehe Tab. 2). Höhere Konsumstufen werden hier mit $1,9 \%$ für regelmäßigen und 1,8\% für schädlichen Alkoholkonsum noch relativ selten berichtet. Hinsichtlich Nikotinkonsums zeigt sich, dass gut ein Fünftel der Personen bereits regelmäßig geraucht hat, und ebenfalls etwa ein Fünftel hat bereits Erfahrung mit dem Konsum von illegalen Drogen.

Inzidenz T0-T2: Über das Follow-up-Intervall (T0-T2) zeigt sich über alle Substanzen hinweg ein bemerkenswerter Anteil an „Neukonsumenten“ bzw. an „Mehrkonsumenten“. Der höchste Zuwachs zeigt sich dabei für gelegentlichen Alkoholkonsum: Mehr als 60\% der Probanden, die zu T0 noch nie bzw. äußerst selten Alkohol getrunken haben, berichten zwischen T0 und T2 erstmalig von gelegentlichem Alkoholkonsum. Nahezu jeder Zehnte berichtet zwischen T0 und T2 von erstmaligem regelmäßigen $(7,6 \%)$ oder schädlichem $(8,8 \%)$ Alkoholkonsum. Hinsichtlich Nikotinkonsum zeigt sich, dass ungefähr ein Drittel (31,8\%) der Personen, die bis zu T0 noch nie bzw. nie regelmäßig geraucht hatten, nun regelmäßig rauchen. Bezüglich des Konsums illegaler Drogen zeigt sich, dass etwa $15 \%$ der Nichtkonsumenten zu T0 zwischen T0 und T2 einen gelegentlichen, d.h. ein- bis viermaligen, Drogenkonsum entwickeln. Etwa ein Viertel (23,8\%) der zu T0 als Nicht- oder Gelegenheitskonsumenten klassifizierten Personen berichtet nun erstmalig von einem regelmäßigen Gebrauch illegaler Drogen.

Kumulierte Lifetime-Inzidenz zu T2: Betrachtet man nun zum Zeitpunkt T2 die „neue“ Lifetime-Prävalenz (kumulierte Lifetime-Inzidenz T2), so zeigt sich, dass mehr als $80 \%$ der inzwischen 18- bis 21-Jährigen mindestens gelegentlich Alkohol konsumieren. Regelmäßiger Alkoholkonsum wird von $7 \%$ der Befragten berichtet und fast jeder Zehnte $(8,3 \%)$ berichtet, bereits schädliche Mengen an Alkohol konsumiert zu haben. Nahezu die Hälfte (45,6\%) der Jugendlichen erfüllt nun die Kriterien für regelmäßiges Rauchen. Ebenfalls nahezu die Hälfte der Jugendlichen weist bis zu diesem Zeitpunkt Erfahrungen mit dem Konsum illegaler Drogen auf, wobei hier häufiger von regelmäßigem $(28,4 \%)$ als von gelegentlichem Konsum (17,3\%) berichtet wird.

\section{DSM-IV-Missbrauch und Abhängigkeit}

Tab. 2 ist ebenfalls zu entnehmen, dass zu T0 die Nikotinabhängigkeit mit 11,2\% die häufigste Substanzdiagnose in der untersuchten Altersgruppe ist. Die Kriterien von Alkoholmissbrauch erfüllen $5,1 \%$ und die einer Alkoholabhängigkeit 1,8\% der befragten Jugendlichen. Eine Störung durch illegale Drogen (Missbrauch oder Abhängigkeit) wird zu diesem Zeitpunkt mit 2,1\% noch relativ selten berichtet. Zwischen T0 und T2 berichtet ein beachtlicher Anteil an Jugendlichen erstmalig von Alkoholmissbrauch (16,8\%). Aber auch Alkoholabhängig- 
Tab. 2 Lifetime-Prävalenz zu T0, Inzidenz von T0 zu T2 und kumulierte Lifetime-Inzidenz zu T2 für Konsum, DSM-IV-Missbrauch und Abhängigkeit von legalen und illegalen Drogen $(n=917)$

\begin{tabular}{|c|c|c|c|c|c|c|c|c|c|}
\hline & \multicolumn{3}{|c|}{$\begin{array}{l}\text { Lifetime-Prävalenz } \\
\text { T0 }\end{array}$} & \multicolumn{3}{|c|}{ Inzidenz von T0 zu T2 } & \multicolumn{3}{|c|}{$\begin{array}{l}\text { Kumulierte Lifetime-Inzidenz } \\
\text { T2 }\end{array}$} \\
\hline & $\mathrm{n}$ & $\% w$ & $(95 \% \mathrm{KI})$ & $\mathrm{n}$ & $\% \mathrm{w}$ & $(95 \% \mathrm{KI})$ & $\mathrm{n}$ & $\% \mathrm{w}$ & $(95 \% \mathrm{KI})$ \\
\hline \multicolumn{10}{|l|}{ Konsum } \\
\hline \multicolumn{10}{|l|}{ Alkohol } \\
\hline kein/seltener Konsum & 677 & 67,6 & $(64,1-71,0)$ & & & & & & \\
\hline gelegentlicher Konsum & 217 & 28,7 & $(25,5-32,2)$ & 454 & 61,7 & $(57,8-65,5)$ & 628 & 68,7 & $(65,3-71,8)$ \\
\hline regelmäßiger Konsum & 11 & 1,9 & $(1,0-3,4)$ & 51 & 7,6 & $(5,7-10,0)$ & 59 & 7,0 & $(5,4-9,1)$ \\
\hline schädlicher Konsum & 12 & 1,8 & $(1,0-3,3)$ & 54 & 8,8 & $(6,6-11,7)$ & 66 & 8,3 & $(6,4-10,7)$ \\
\hline \multicolumn{10}{|l|}{ Nikotin } \\
\hline kein/seltener Konsum & 757 & 79,8 & $(76,7-82,7)$ & & & & & & \\
\hline regelmäßiger Konsum & 160 & 20,2 & $(17,4-23,3)$ & 256 & 31,8 & $(28,4-35,4)$ & 416 & 45,6 & $(42,1-49,1)$ \\
\hline \multicolumn{10}{|l|}{ illegale Drogen } \\
\hline kein Konsum & 767 & 81,6 & $(78,5-84,2)$ & & & & & & \\
\hline gelegentlicher Konsum (1- bis 4-mal) & 95 & 11,0 & $(8,9-13,4)$ & 120 & 14,2 & $(11,9-17,0)$ & 159 & 17,3 & $(14,8-20,1)$ \\
\hline regelmäßiger Konsum (5+) & 55 & 7,5 & $(5,7-9,8)$ & 194 & 23,8 & $(20,8-27,1)$ & 249 & 28,4 & $(25,3-31,7)$ \\
\hline \multicolumn{10}{|l|}{ DSM-IV-Missbrauch und Abhängigkeit } \\
\hline \multicolumn{10}{|l|}{ Alkohol } \\
\hline weder Missbrauch noch Abhängigkeit & 870 & 93,2 & $(90,9-94,9)$ & & & & & & \\
\hline Missbrauch (ohne Abhängigkeit) & 34 & 5,1 & $(3,6-7,1)$ & 153 & 16,8 & $(14,4-19,6)$ & 182 & 20,1 & $(17,4-23,1)$ \\
\hline Abhängigkeit & 13 & 1,8 & $(1,0-3,1)$ & 48 & 5,4 & $(4,0-7,3)$ & 61 & 6,8 & $(5,2-8,9)$ \\
\hline \multicolumn{10}{|l|}{ Nikotin } \\
\hline keine Abhängigkeit & 832 & 88,8 & $(86,1-91,0)$ & & & & & & \\
\hline Abhängigkeit & 85 & 11,2 & $(9,1-13,9)$ & 125 & 13,9 & $(11,7-16,5)$ & 210 & 23,6 & $(20,7-26,7)$ \\
\hline \multicolumn{10}{|l|}{ Drogen } \\
\hline weder Missbrauch noch Abhängigkeit & 899 & 97,9 & $(96,6-98,7)$ & & & & & & \\
\hline Missbrauch oder Abhängigkeit & 18 & 2,1 & $(1,3-3,4)$ & 69 & 8,3 & $(6,5-10,6)$ & 87 & 10,2 & $(8,2-12,7)$ \\
\hline
\end{tabular}

Anmerkungen: $\mathrm{n}=$ ungewichtete Personenzahl; \%w = gewichteter Prozentanteil; KI= Konfidenzintervall; Definition der Konsumkategorien: Alkohol: nie/seltener Konsum: nie mehr als 11-mal innerhalb von 12 Monaten Alkohol konsumiert; gelegentlicher Konsum: mindestens 12-mal pro Jahr, aber nie mehr als dreimal pro Monat; regelmäßiger Konsum: mindestens viermal pro Monat, aber nie schädlicher Konsum; schädlicher Konsum: in der Periode des stärksten Konsums Frauen mindestens $20 \mathrm{~g}$ und Männer mindestens $40 \mathrm{~g}$ Alkohol pro Tag. Nikotin: kein/seltener Konsum: nie geraucht oder nie innerhalb von 4 Wochen täglich geraucht; regelmäßiger Konsum: mindestens 4 Wochen täglich geraucht; Referenzgruppen zur Berechnung der Inzidenzraten: Alkohol: Inzidenz von gelegentlichem Konsum: alle Nichtkonsumenten und Konsumenten mit seltenem Konsum zu T0; Inzidenz von regelmäßigem Konsum: alle Probanden, die zu T0 weder regelmäßigen noch schädlichen Konsum berichteten; Inzidenz von schädlichem Konsum: alle Probanden, die zu T0 keinen schädlichen Konsum aufwiesen; Inzidenz von Missbrauch: alle Probanden ohne eine Diagnose zu T0; Inzidenz von Abhängigkeit: alle Probanden ohne eine Abhängigkeitsdiagnose zu T0; Nikotin: Inzidenz von regelmäßigem Konsum: alle Probanden, welche zu T0 nicht die Kriterien von regelmäßigem Konsum erfüllten; Inzidenz von Abhängigkeit: alle Probanden ohne eine Abhängigkeitsdiagnose zu T0; eine Missbrauchsdiagnose wird hier nicht gestellt; illegale Drogen: Inzidenz von gelegentlichem Konsum: alle Nichtkonsumenten zu T0; Inzidenz von regelmäßigem Konsum: alle Nicht- und gelegentlichen Konsumenten zu T0; Inzidenz von Missbrauch oder Abhängigkeit: alle Probanden ohne eine Missbrauchs- oder Abhängigkeitsdiagnose zu T0;

keit (5,4\%) und Missbrauch oder Abhängigkeit durch illegale Substanzen $(8,3 \%)$ werden im Vergleich zu den LifetimePrävalenzraten zu T0 von einem bemerkenswerten Anteil von Jugendlichen erstmalig berichtet. 13,9\% der Jugendlichen, die zu T0 noch keine Nikotinabhängigkeit aufwiesen, entwickeln diese während des Follow-up-Intervalls. Aufgrund der hohen Inzidenzraten im Intervall $\mathrm{T} 0-\mathrm{T} 2$ liegen die kumulierten Lifetime-Inzidenzen für DSM-IV-Missbrauch und -Abhängigkeit zu T2 nun deutlich über den zu T0 ermittelten LifetimePrävalenzen. So erfüllt zum Zeitpunkt T2 nun jede fünfte Person lebenszeitbezogen die Kriterien für Alkoholmissbrauch und 6,8\% die Kriterien der Alkoholabhängigkeit. Störungen durch illegale Drogen werden, bezogen auf die Lebenszeit, mit 10,2\% ebenfalls etwa fünfmal häufiger berichtet als zu T0, während sich die Raten für Nikotinabhängigkeit etwa verdoppelt haben (23,6\% zu T2 gegenüber $11,2 \%$ zu T0).

\section{Elterliche Alkoholbelastung und Substanzkonsum bei den Kindern}

Werden nun die Konsummuster für Alkohol, Nikotin und illegale Drogen getrennt danach betrachtet, ob eine elterliche Alkoholbelastung vorliegt oder nicht, so lässt sich aus Tab. 3 zunächst für den Konsum von Alkohol entnehmen, dass Probanden mit einer elterlichen Alkoholbelastung im Vergleich zu Probanden ohne eine elterliche Alkoholbelastung mit höheren Konsumkategorien steigende Raten aufweisen. Über alle untersuchten Kategorien hinweg neigen Kinder alkoholbelasteter Eltern zu höheren Konsumkategorien $(C U M O R=1,5)$. Ähnliche Ergebnisse zeigen sich ebenfalls für den Konsum von Nikotin und illegalen Drogen: Probanden mit einer elterlichen Alkoholbelastung berichten deutlich höhere Raten in den jeweils höheren Konsumkategorien. Für keine Substanz zeigen sich signifikante Interaktionen zwischen dem Geschlecht der Probanden und elterlicher Alkoholbelastung, d.h. die Assoziationen sind vergleichbar hoch für Söhne und Töchter alkoholbelasteter Eltern.

\section{Elterliche Alkoholbelastung und DSM-IV-Missbrauch und Abhängigkeit von legalen und illegalen Drogen bei den Kindern}

Tab. 4 zeigt, dass Probanden mit einer elterlichen Alkoholbelastung im Vergleich zu Probanden ohne elterliche Alkoholbelastung sowohl höhere Raten an Alkoholmissbrauch (24,2 vs. $17,8 \%$; OR $=1,8$ ) als auch höhere Raten an Alkoholabhängigkeit $(9,7$ vs. $5,2 \%$; OR $=2,0)$ berichten. Höhere Raten werden 
Tab. 3 Assoziationen zwischen elterlicher Alkoholbelastung und Konsum legaler und illegaler Substanzen bei Jugendlichen

\begin{tabular}{|c|c|c|c|c|c|c|c|c|c|}
\hline \multirow[b]{2}{*}{ Substanz } & \multicolumn{3}{|c|}{$\begin{array}{c}\text { nein } \\
(\mathrm{Nw}=573)\end{array}$} & \multicolumn{3}{|c|}{$(\mathrm{Nw}=329)$} & \multicolumn{3}{|c|}{ Assoziationen } \\
\hline & $\mathrm{Nw}$ & $\% w$ & $(95 \% \mathrm{KI})$ & Nw & $\% w$ & $(95 \% \mathrm{KI})$ & $\begin{array}{l}\text { CUM } \\
\text { OR }\end{array}$ & & $(95 \% \mathrm{KI})$ \\
\hline \multicolumn{10}{|l|}{ Alkohol } \\
\hline nie/selten & 99 & 17,2 & $(14,3-20,6)$ & 46 & 13,9 & $(10,5-18,2)$ & & & \\
\hline gelegentlich & 401 & 70,0 & $(65,9-73,8)$ & 218 & 66,4 & $(60,6-71,7)$ & & & \\
\hline regelmäßig & 38 & 6,6 & $(4,7-9,3)$ & 25 & 7,7 & $(5,1-11,5)$ & & & \\
\hline schädlich & 35 & 6,2 & $(4,3-8,8)$ & 39 & 12,0 & $(8,4-16,9)$ & 1,5 & $*$ & $(1,1-2,1)$ \\
\hline \multicolumn{10}{|l|}{ Nikotin } \\
\hline nie/selten & 336 & 58,7 & $(54,4-63,0)$ & 154 & 47,0 & $(41,2-52,8)$ & & & \\
\hline regelmäßig & 236 & 41,3 & $(37,1-45,6)$ & 174 & 53,1 & $(47,2-58,8)$ & 1,6 & * & $(1,2-2,2)$ \\
\hline \multicolumn{10}{|l|}{ illegale Drogen } \\
\hline nie & 339 & 59,2 & $(54,8-63,4)$ & 143 & 43,5 & $(37,9-49,4)$ & & & \\
\hline gelegentlich (1- bis 4-mal) & 68 & 11,9 & $(9,4-15,1)$ & 39 & 12,0 & $(8,6-16,4)$ & & & \\
\hline regelmäßig $(5+)$ & 165 & 28,9 & $(25,0-33,0)$ & 146 & 44,5 & $(38,8-50,4)$ & 2,0 & * & $(1,5-2,6)$ \\
\hline
\end{tabular}

Anmerkungen: Nw zeigt die gewichtete Anzahl an; \%w die gewichteten Prozente; KI= Konfidenzintervall; OR = Odds Ratio; CUMOR = Kumulativer Odds Ratio; kontrolliert nach Alter und Geschlecht der Probanden; * $\mathrm{p}<0.05$;

von ihnen jedoch ebenfalls hinsichtlich Nikotinabhängigkeit (32,8 vs. $18,3 \%$; OR = 2,2) sowie Missbrauch und Abhängigkeit von illegalen Drogen $(14,4$ vs. $7,8 \%$; OR $=2,1)$ berichtet. Auch hier zeigen sich über alle betrachteten Substanzstörungen hinweg keine signifikanten Interaktionen zwischen dem Geschlecht der Probanden und elterlicher Alkoholbelastung.

\section{Elterliche Alkoholbelastung und Übergang in bestimmte Stadien des Konsums von Alkohol bei den Kindern}

Wie Tab. 5 zu entnehmen ist, zeigt sich eine signifikante Assoziation zwischen elterlicher Alkoholbelastung und der Progression von gelegentlichem zu regelmäßigem Alkoholkonsum bei den Kindern ( $C O R=1,7)$. Detailanalysen konnten aufzeigen, dass hier im Vergleich zu Probanden ohne elterli- che Alkoholbelastung speziell Probanden mit zwei betroffenen Eltern ein höheres Risiko für den Übergang vom gelegentlichen in den regelmäßigen Konsum aufweisen $(C O R=2,9$; $95 \% \mathrm{KI}=1,4-6,0)$.

\section{Elterliche Alkoholbelastung und Erstauftreten von Substanzkonsum und Substanzstörungen}

Abb. 1 zeigt getrennt für Probanden mit und ohne elterliche Alkoholbelastung für jedes Lebensalter die geschätzte altersspezifische Lebenszeit-Inzidenz für schädlichen Alkoholkonsum (A), regelmäßigen Nikotinkonsum (B) und erstmaligen Drogenkonsum (C), d.h. die geschätzte Wahrscheinlichkeit, mit welcher bis zu einem bestimmten Alter jemals Alkohol in

Tab. 4 Assoziationen zwischen elterlicher Alkoholbelastung und DSM-IV-Missbrauch und Abhängigkeit (kumulierte Lifetime-Inzidenz zu T2) bei den Jugendlichen

\begin{tabular}{|c|c|c|c|c|c|c|c|c|c|}
\hline \multirow[b]{2}{*}{ Substanzstörung } & \multirow[b]{2}{*}{ Nw } & \multicolumn{2}{|c|}{$\begin{array}{c}\text { nein } \\
(\mathrm{Nw}=573)\end{array}$} & \multicolumn{3}{|c|}{$\begin{array}{c}\mathrm{ja} \\
(\mathrm{Nw}=329)\end{array}$} & \multicolumn{3}{|c|}{ Assoziationen } \\
\hline & & $\% w$ & $(95 \% \mathrm{KI})$ & Nw & $\% w$ & $(95 \% \mathrm{KI})$ & \multicolumn{2}{|l|}{ OR } & $(95 \% \mathrm{KI})$ \\
\hline \multicolumn{10}{|l|}{ Alkohol } \\
\hline keine Diagnose & 441 & 77,1 & $(73,1-80,6)$ & 217 & 66,1 & $(60,4-71,4)$ & & & \\
\hline Missbrauch (ohne Abhängigkeit) ${ }^{1}$ & 102 & 17,8 & $(14,6-21,4)$ & 80 & 24,2 & $(19,6-29,5)$ & 1,8 & $*$ & $(1,2-2,6)$ \\
\hline Abhängigkeit ${ }^{2}$ & 30 & 5,2 & $(3,6-7,5)$ & 32 & 9,7 & $(6,6-13,9)$ & 2,0 & $*$ & $(1,1-3,6)$ \\
\hline \multicolumn{10}{|l|}{ Nikotin } \\
\hline keine Diagnose & 468 & 81,7 & $(78,1-84,8)$ & 221 & 67,2 & $(61,4-72,5)$ & & & \\
\hline Abhängigkeit ${ }^{3}$ & 105 & 18,3 & $(15,2-22,0)$ & 108 & 32,8 & $(27,5-38,6)$ & 2,2 & $*$ & $(1,5-3,1)$ \\
\hline \multicolumn{10}{|l|}{ illegale Drogen } \\
\hline keine Diagnose & 528 & 92,2 & $(89,3-94,3)$ & 281 & 85,6 & $(80,8-89,3)$ & & & \\
\hline Missbrauch oder Abhängigkeit ${ }^{4}$ & 45 & 7,8 & $(5,7-10,7)$ & 47 & 14,4 & $(10,7-19,2)$ & 2,1 & $*$ & $(1,3-3,4)$ \\
\hline
\end{tabular}

Anmerkungen: Nw zeigt die gewichtete Anzahl an; \%w die gewichteten Prozente; KI = Konfidenzintervall; OR = Odds Ratio; kontrolliert nach Alter und Geschlecht der Probanden; * $\mathrm{p}<0,05$,

${ }^{1}$ Referenzgruppe sind Probanden ohne eine Alkoholstörung,

2 Referenzgruppe sind Probanden ohne eine Alkoholabhängigkeit,

${ }^{3}$ Referenzgruppe sind Probanden ohne eine Nikotinabhängigkeit,

${ }^{4}$ Referenzgruppe sind Probanden ohne Missbrauch/Abhängigkeit von illegalen Drogen 
Tab. 5 Elterliche Alkoholbelastung und Progression des Alkoholkonsums bei den Jugendlichen $(\mathrm{n}=917)$

\begin{tabular}{|c|c|c|c|c|c|c|c|c|c|c|c|}
\hline \multirow[b]{3}{*}{ elterliche Alkoholbelastung } & \multicolumn{4}{|c|}{ Alkoholkonsum der Jugendlichen } & & & & & & & \\
\hline & \multicolumn{2}{|c|}{$\begin{array}{l}\text { mindestens } \\
\text { gelegentlicher } \\
\text { Konsum } \\
(\mathrm{Nw}=573)\end{array}$} & \multicolumn{2}{|c|}{$\begin{array}{l}\text { mindestens } \\
\text { regulärer } \\
\text { Konsum unter } \\
\text { gelegentlichen } \\
\text { Konsumenten } \\
(\mathrm{Nw}=138)\end{array}$} & \multicolumn{2}{|c|}{$\begin{array}{l}\text { mindestens } \\
\text { schädlicher } \\
\text { Konsum unter } \\
\text { regulären } \\
\text { Konsumenten } \\
(\mathrm{Nw}=75)\end{array}$} & \multicolumn{2}{|c|}{$\begin{array}{l}\text { Progression } \\
\text { in } \\
\text { gelegentlichen } \\
\text { Konsum }\end{array}$} & \multicolumn{2}{|c|}{$\begin{array}{l}\text { Progression } \\
\text { in } \\
\text { regelmäßigen } \\
\text { Konsum }\end{array}$} & $\begin{array}{l}\text { Progression } \\
\text { in } \\
\text { schädlichen } \\
\text { Konsum }\end{array}$ \\
\hline & $\mathrm{Nw}$ & $\% w$ & $\mathrm{Nw}$ & $\% w$ & $\mathrm{Nw}$ & $\% w$ & COR & $(95 \% \mathrm{KI})$ & COR & $(95 \% \mathrm{KI})$ & COR $(95 \% \mathrm{KI})$ \\
\hline $\begin{array}{l}\text { keine elterliche Belastung } \\
\qquad(\mathrm{Nw}=573)\end{array}$ & 474 & 82,8 & 73 & 15,5 & 35 & 48,2 & & & & & \\
\hline $\begin{array}{l}\text { elterliche Belastung } \\
\qquad(\mathrm{Nw}=329)\end{array}$ & 283 & 86,1 & 65 & 22,9 & 39 & 61,0 & 1,3 & $(0,8-1,9)$ & 1,7 & $*(1,1-2,7)$ & $(0,8-3,9)$ \\
\hline
\end{tabular}

Anmerkungen: Nw zeigt die gewichtete Anzahl an; \%w die gewichteten Prozente; KI = Konfidenzintervall; COR = Continuation Odds Ratio; kontrolliert nach Alter und Geschlecht der Probanden; ${ }^{*} \mathrm{p}<0,05$

schädlichen Mengen, jemals regelmäßig Nikotin und jemals illegale Drogen konsumiert werden.

Die Kurven zeigen zunächst für schädlichen Alkoholkonsum, dass sowohl von Jugendlichen mit als auch von Jugendlichen ohne elterliche Alkoholbelastung schädliche Mengen von Alkohol ab dem Alter von 14/15 Jahren konsumiert werden. Der Verlauf der beiden Kurven weist jedoch darauf hin, dass Probanden mit einer elterlichen Alkoholbelastung über die untersuchte Altersspanne hinweg in jedem Lebensalter höhere Lebenszeit-Inzidenzraten berichten, d.h. eine höhere Wahrscheinlichkeit aufweisen, jemals schädliche Mengen von Alkohol zu konsumieren ( $\mathrm{HR}=2,0 ; 95 \% \mathrm{KI}=1,1-3,4)$. In Detailanalysen prüften wir, ob Jugendliche mit einer elterlichen Alkoholbelastung auch früher als Probanden ohne elterliche Alkoholbelastung schädliche Mengen von Alkohol konsumieren. Hier fanden wir, dass speziell Jugendliche mit zwei betroffenen Eltern einen früheren Einstieg aufweisen als Probanden ohne elterliche Alkoholbelastung (HR Alter*beide Eltern betroffen $=0,6 ; 95 \% \mathrm{KI}=0,3-0,9)$. Kein Unterschied zeigte sich hingegen zwischen Jugendlichen mit einem betroffenen Elternteil und Jugendlichen ohne elterliche Alkoholbelastung (HR Alter $*$ ein Elternteil betroffen $=1,0 ; 95 \%$ $\mathrm{KI}=0,7-1,4)$. Bei einer Betrachtung der Kurven für erstmaliges regelmäßiges Rauchen zeigt sich, dass Jugendliche mit oder ohne elterliche Alkoholbelastung etwa ab 12/13 Jahren erstmalig regelmäßig rauchen. Die Kurve steigt in beiden Gruppen bis in das Alter von 20 Jahren kontinuierlich an, jedoch mit höheren Inzidenzraten in der Gruppe der Jugendlichen mit elterlicher Alkoholbelastung ( $H R=1,4 ; 95 \%$ $\mathrm{KI}=1,2-1,8)$. Auch hier ergaben Detailanalysen, dass speziell Jugendliche mit zwei betroffenen Eltern einen früheren Einstieg in den regelmäßigen Nikotinkonsum zeigen als Jugendliche ohne Alkoholbelastung (HR Alter*beide Eltern betroffen $=0,7 ; 95 \% \mathrm{KI}=0,5-0,8)$. Bezüglich des Erstkonsums von illegalen Drogen lässt sich aus Abb. 1 entnehmen, dass sich dieser wiederum in beiden Gruppen ab dem 11./12. Lebensjahr beobachten lässt. Die Kurven steigen in beiden Gruppen nahezu kontinuierlich bis zum 20. Lebensjahr an und flachen dann ab. Auch hier berichten Jugendliche mit elterlicher Alkoholbelastung höhere Inzidenzraten als Jugendliche ohne elterliche Alkoholbelastung ( $\mathrm{HR}=1,6 ; 95 \% \mathrm{KI}=1,3-1,9)$. Für den Erstkonsum illegaler Drogen zeigt sich keine Interaktion mit dem Alter der Probanden.
Hinsichtlich des Erstauftretens von Alkoholmissbrauch und -abhängigkeit, Nikotinabhängigkeit sowie Missbrauch/Abhängigkeit von illegalen Drogen (siehe Abb. 2) zeigen unsere Befunde, dass für alle der genannten substanzbedingten Störungen Probanden mit elterlicher Alkoholbelastung über jedes untersuchte Lebensalter hinweg höhere Inzidenzraten aufweisen als Probanden ohne elterliche Alkoholbelastung. Es konnten keine signifikanten Interaktionen mit dem Alter der Probanden ermittelt werden, d.h. das Einstiegsalter war vergleichbar für Probanden mit und ohne elterliche Alkoholbelastung.

\section{Diskussion}

Basierend auf einer prospektiven epidemiologischen Längsschnittuntersuchung wurden in dem vorliegenden Beitrag Befunde zur Frage vorgelegt, inwiefern eine elterliche Alkoholbelastung mit der Entwicklung von Suchtproblemen bei ihren Kindern assoziiert ist. Die hier vorgestellten Ergebnisse ergänzen bisher vorliegende Forschungsbefunde zu diesem Forschungsgebiet insofern, als

1. diese an einer repräsentativen Stichprobe von Jugendlichen ermittelt wurden, daher eine höhere Generalisierbarkeit als Ergebnisse aus klinischen Studien gewährleisten,

2. der Studie ein prospektiv-longitudinales Design zugrunde liegt, welches eine zeitnahe Erfassung wesentlicher Verlaufsaspekte, wie etwa des Erstkonsumalters, gewährleistet,

3. durch die Erhebungsmethodik mittels persönlicher Interviews sowie spezieller befragungs- und auswertungstechnischer Merkmale des standardisierten computerisierten Instrumentes, des M-CIDI, eine hohe Reliabilität, Validität und Auswertungsobjektivität erreicht wurde,

4. Missbrauch und Abhängigkeit nach den strikten Kriterien des DSM-IV erhoben wurden,

5. die Zielpersonen über die Risikoperiode des initialen Gebrauchs von legalen und illegalen Drogen hinweg untersucht wurden, sowie

6. für eine repräsentative Stichprobe direkte Elterninformationen erhoben wurden.

Trotz all dieser Stärken unserer Studie sollen jedoch auch methodische Einschränkungen genannt werden, auf deren Hintergrund die Befunde bewertet werden müssen. So konnte 


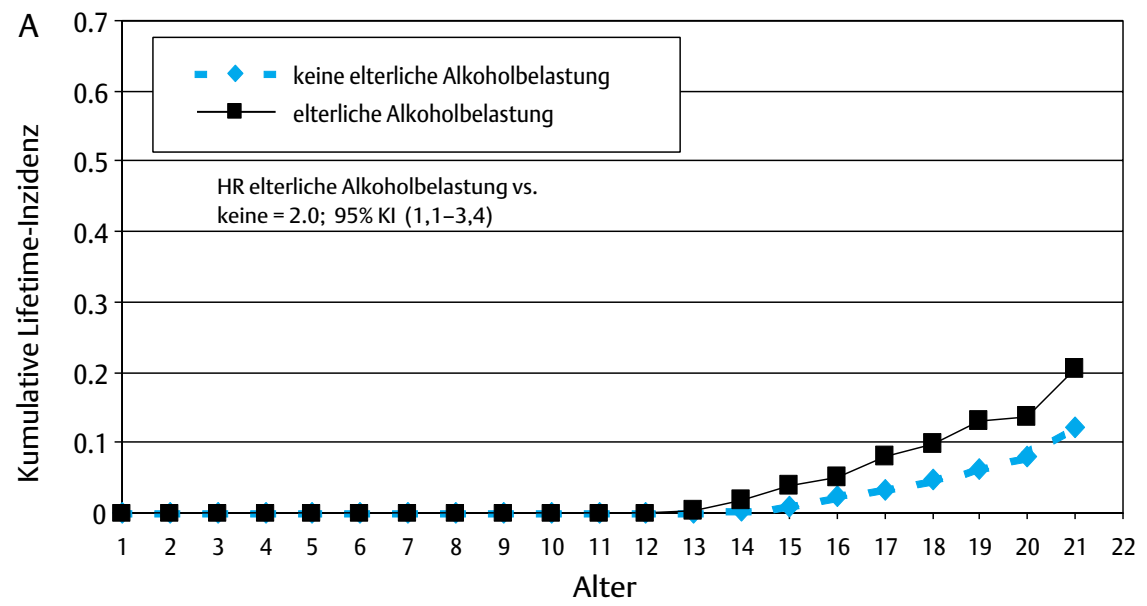

Abb. 1 Erstauftretensalter von A schädlichem Alkoholkonsum, B regelmäßigem Rauchen und

C Konsum illegaler Drogen nach elterlicher Alkoholbelastung.
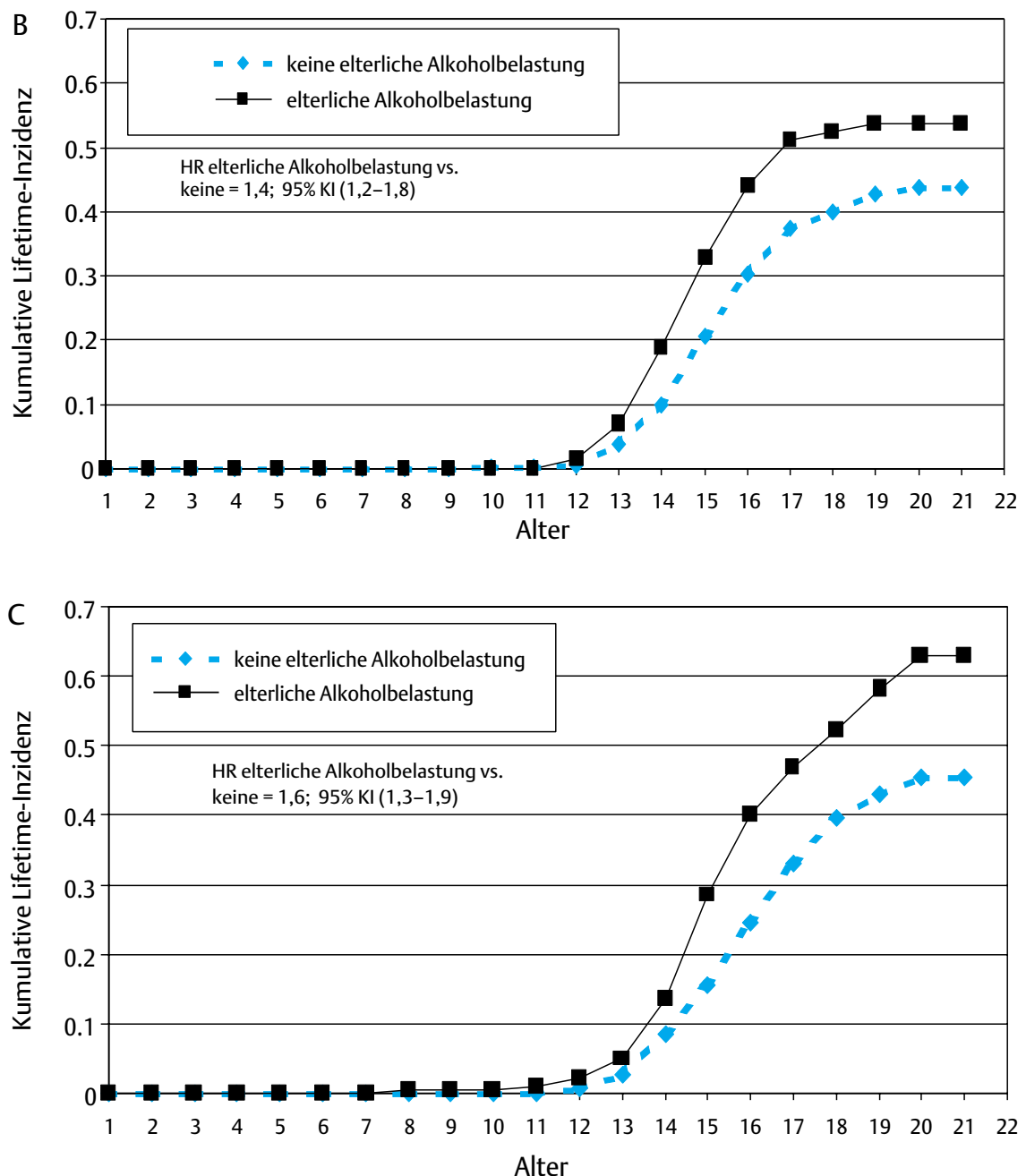

zur Erfassung elterlicher Psychopathologie zumindest für die Väter lediglich indirekte „family-history“-Information erhoben werden, d.h. es liegen keine direkten diagnostischen Informationen für sie vor. Indirekte diagnostische Information könnte vom diagnostischen Status der befragten Personen beeinflusst sein, etwa in dem Sinne, dass Personen mit einem Alkoholproblem solche auch besser bei Familienangehörigen erkennen [42, 43]. Diese Möglichkeit prüften wir durch einen Vergleich der Sensitivität der family-historyInformationen zwischen selbst betroffenen und nicht selbst betroffenen Informanden und den an den Müttern direkt erhobenen diagnostischen Informationen. Wir fanden keinen Hinweis darauf, dass Jugendliche mit einer Alkoholstörung eine Alkoholbelastung bei ihren Müttern besser erkennen und es gab somit keinen Hinweis auf eine Verzerrung der indirekten diagnostischen Information durch den diagnosti- 


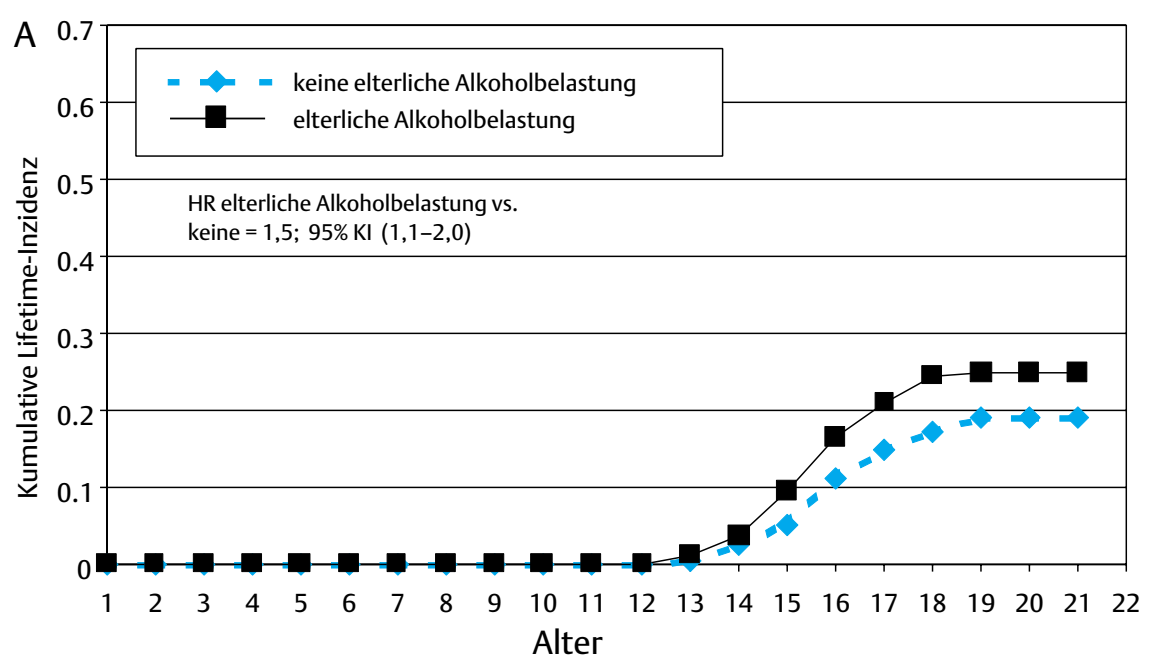

Abb. 2 Erstauftretensalter von

A Alkoholabhängigkeit,

B Nikotinabhängigkeit und

C Missbrauch/Abhängigkeit von illegalen

Drogen nach elterlicher Alkoholbelastung.
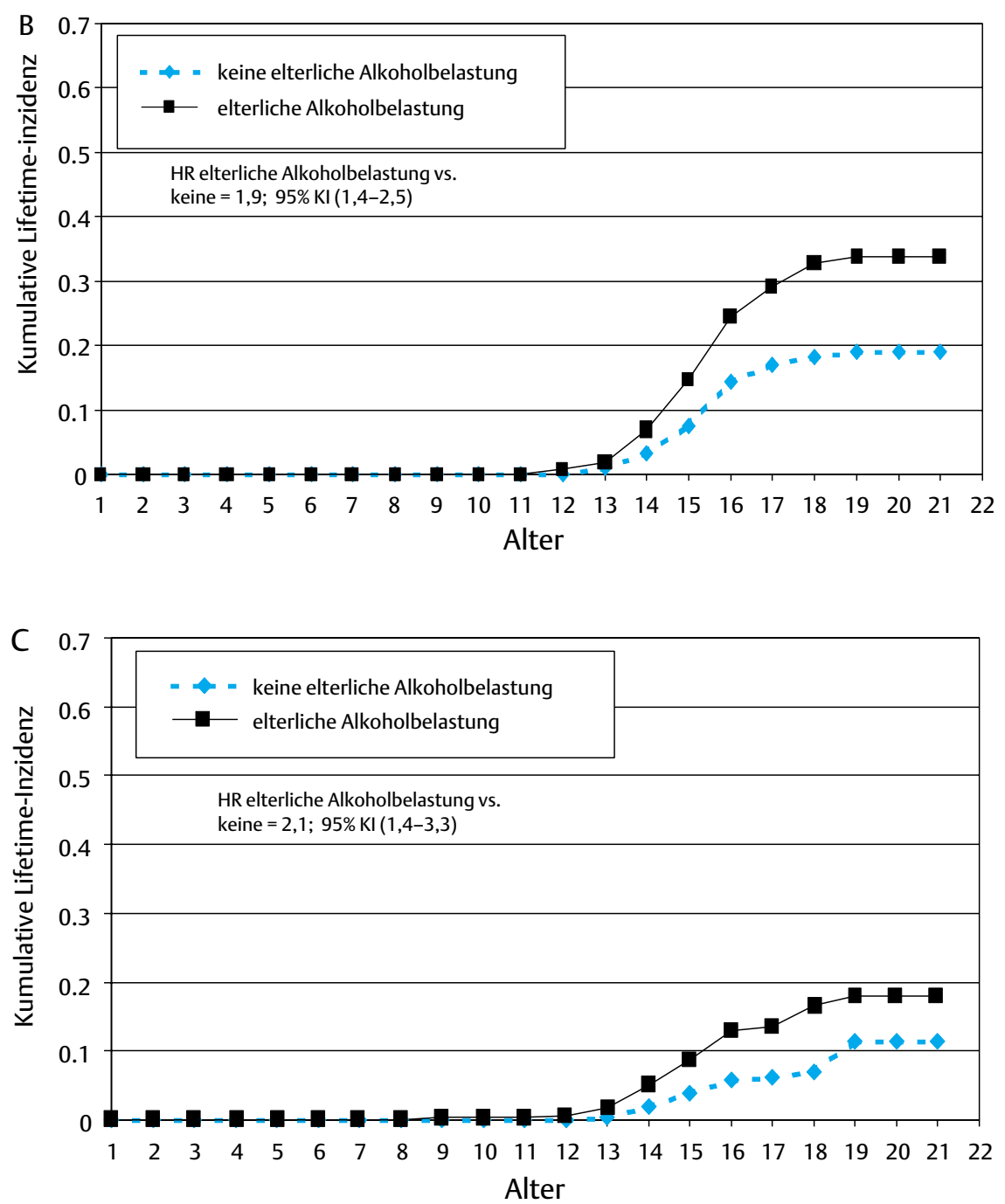

schen Status der Jugendlichen. Eine weitere methodische Einschränkung bezieht sich auf den Umstand, dass die Altersspanne der untersuchten Stichprobe auf 21 Jahre begrenzt ist, was bedeutet, dass noch nicht alle untersuchten Jugendlichen die Hochrisikozeit für die Entwicklung von Konsum, Miss- brauch und Abhängigkeit von psychotropen Substanzen passiert haben. Dafür sprechen zum einen Befunde aus der Basisuntersuchung, die sich auf die Gesamtstichprobe der damals 14- bis 24-Jährigen beziehen und die v.a. für illegale Drogen und Alkohol, weniger ausgeprägt für Nikotin, kon- 
tinuierlich hohe Inzidenzraten für Substanzkonsum und -störungen bis an die obere Altersgrenze, d.h. bis in die Mitte der dritten Lebensdekade, ergaben $[2,31,32]$. Auch die in dieser Arbeit berichteten hohen Inzidenzraten für Konsum und Störungen durch Alkohol und illegale Drogen zwischen T0 und T2 weisen darauf hin, dass die Hochrisikozeit in der untersuchten Altersspanne noch nicht abgeschlossen ist. Um die Zahl sog. „falschnegativer“ Fälle zu minimieren, zogen wir zur Analyse der familiären Assoziationsmuster die kumulierten Lebenszeit-Inzidenzen zu T2 heran, welche die maximale diagnostische Information, die für die Jugendlichen im Verlauf der Studie erfasst werden konnte, einschließen.

\section{Lebenszeitprävalenzen und 4-Jahres-Inzidenzen von Substanzkonsum, -missbrauch und -abhängigkeit}

Wie wir auch in bereits vorliegenden Publikationen $\mathrm{zu}$ Befunden der EDSP-Basisuntersuchung bzw. ausgewählten Follow-up-Befunden beschrieben haben [31-33, 44], waren zum Zeitpunkt der Basisuntersuchung Konsum, Missbrauch und Abhängigkeit sowohl von legalen als auch illegalen Substanzen in den untersuchten Altersgruppen noch relativ gering ausgeprägt. Unsere Ergebnisse zeigen jedoch, dass über das 4-Jahres-Follow-up-Intervall hinweg Konsum sowie substanzbezogener Missbrauch und Abhängigkeit merklich zunehmen. Unsere Befunde ergeben ferner, dass Alkohol und Nikotin als das größte Problem $\mathrm{zu}$ sehen sind. Sowohl bezüglich der T0-T2-Inzidenzraten als auch der kumulierten Lifetime-Inzidenzraten zu T2 überschreiten die Konsum-, Missbrauchs- und Abhängigkeitsraten um ein Vielfaches die Raten von Konsum und Missbrauch/Abhängigkeit illegaler Drogen.

\section{Elterliche Alkoholbelastung und Alkoholkonsum, -missbrauch und -abhängigkeit}

Vergleichbar zu bisher vorliegenden Befunden [5, 7, 10, 12, 17] konnten wir zunächst bestätigen, dass elterliche Alkoholbelastung bereits im Jugendalter mit einem erhöhten Risiko verbunden ist, größere und auch schädlichere Mengen an Alkohol zu konsumieren. So berichten doppelt so viele Jugendliche mit einer elterlichen Alkoholbelastung als Jugendliche ohne eine solche Belastung, bereits schädliche Mengen an Alkohol konsumiert zu haben. Durch die detaillierte Analyse von Übergängen in bestimmte Konsumstadien konnten wir erstmals nachweisen, dass elterliche Alkoholbelastung speziell mit dem Übergang vom gelegentlichen in den regelmäßigen Alkoholkonsum assoziiert ist. Elterliche Alkoholbelastung stellt nach diesen Befunden somit einen Risikofaktor sowohl für das Konsumieren von größeren oder gar schädlichen Mengen von Alkohol wie auch für die Stabilisierung des Trinkverhaltens während der Adoleszenz dar. Auch die ermittelten familiären Assoziationen zwischen elterlicher Alkoholbelastung und alkoholbedingtem Missbrauch und Abhängigkeit entsprechen weitestgehend Befunden, wie sie in der Regel an klinischen Stichproben ermittelt wurden [7, 9, $10,12]$ und bestätigen diese familiäre Belastung als bedeutsamen Prädiktor für die Entwicklung von Missbrauchs- und Abhängigkeitssyndromen. Speziell für Alkoholabhängigkeit konnten wir zeigen, dass Alkoholabhängigkeit unter Jugendlichen mit einer elterlichen Alkoholbelastung etwa doppelt so häufig diagnostiziert wird wie unter Jugendlichen ohne eine elterliche Belastung. Bis heute kann nicht genau erklärt werden, welche Mechanismen für die familiäre Transmission von alkoholbedingten Problemen (oder Störungen) verantwortlich sind. Aufgrund bisheriger Forschungsbefunde ist sehr wahrscheinlich davon auszugehen, dass sowohl genetische Faktoren als auch familiäre Umgebungsfaktoren an der familiären Übertragung beteiligt sind [45]. Welche spezifischen Faktoren an der Übertragung beteiligt sind und in welcher Art und Weise sie miteinander interagieren (z.B. Gen-Umwelt-Interaktion), kann jedoch noch längst nicht befriedigend beantwortet werden.

\section{Elterliche Alkoholbelastung und Konsum, Missbrauch und Abhängigkeit von Nikotin und illegalen Drogen}

Eine elterliche Alkoholbelastung erhöht nicht nur das Risiko für einen vermehrten Konsum von Alkohol während der Jugend, sondern ebenfalls für einen höheren Konsum von Nikotin sowie von illegalen Drogen. So berichten Jugendliche mit einer elterlichen Alkoholbelastung zum Zeitpunkt der zweiten Follow-up-Untersuchung signifikant höhere Raten sowohl an regelmäßigem Nikotinkonsum als auch an regelmäßigem Konsum illegaler Drogen. Eine elterliche Alkoholbelastung scheint sich demnach nicht nur auf das Trinkverhalten ihrer Kinder, sondern ebenfalls auf das Konsumieren von anderen psychotropen Substanzen niederzuschlagen [9-11, 46]. Auch für Nikotin und illegale Drogen gilt, dass Jugendliche mit einer elterlichen Alkoholbelastung nicht nur für einen höheren Konsum anfällig sind, sondern dass diese ebenfalls nahezu doppelt so häufig die Missbrauchs- und Abhängigkeitskriterien für diese Substanzen erfüllen. Nach diesen Befunden scheint elterliche Alkoholbelastung somit über die unterschiedlichen Substanzen hinweg das Risiko für Substanzstörungen zu erhöhen $[11,18]$. Obwohl wir die Frage letztendlich nicht beantworten können, könnten diese Ergebnisse möglicherweise darauf hinweisen, dass familiär eine generelle Vulnerabilität für Substanzkonsum übertragen wird [47], während die Präferenz einer bestimmten Substanz eventuell stärker durch das Umfeld determiniert wird.

\section{Geschlechtsspezifität der familiären Assoziationen}

Bisher vorliegende Befunde zur Frage nach geschlechtsspezifischen familiären Assoziationsmustern lassen kein einheitliches Bild zu. So sprechen die Ergebnisse einiger Studien für eine geschlechtsspezifische familiäre Transmission [11, 48], während andere Studien dies nicht nahe legen $[4,13,49]$. Auch unsere Befunde sprechen eher gegen eine geschlechtsspezifische familiäre Assoziation, da wir vergleichbar hohe Assoziationen für männliche und weibliche Jugendliche fanden. Wir prüften zusätzlich, ob sich auf der Basis unserer Daten Interaktionen zwischen dem Geschlecht des betroffenen Elternteils und dem Geschlecht des betroffenen Jugendlichen zeigen, konnten aber keine Hinweise hierfür finden.

\section{Elterliche Alkoholbelastung und Alter bei Beginn}

Unsere Analysen bezüglich des Erstkonsums bekräftigen zunächst unsere bereits an der gesamten EDSP-Stichprobe in der Basisuntersuchung ermittelten Befunde, dass das Jugendalter eine Hochrisikoperiode für den Einstieg in den Substanzkonsum [2, 31, 32] darstellt. So zeigen die Ergebnisse der Survivalanalysen, dass sowohl erstmaliger Konsum von schädlichen Mengen an Alkohol, erstmaliger regelmäßiger 
Nikotinkonsum als auch erstmaliger Konsum von illegalen Drogen etwa mit dem Beginn des Jugendalters einsetzt und dann kontinuierlich über die Jugendzeit hinweg zunimmt. Ab dem erstmaligen Auftreten dieser Konsummengen berichten Jugendliche mit einer elterlichen Alkoholbelastung über die gesamte untersuchte Altersspanne hinweg (d.h. bis 21 Jahre) höhere Lebenszeit-Inzidenzraten, d.h. sie haben in jedem Alter eine höhere Wahrscheinlichkeit als Jugendliche ohne elterliche Belastung, erstmalig schädliche Mengen von Alkohol zu konsumieren, regelmäßig zu rauchen und/oder erstmalig illegale Drogen zu nehmen. Ähnliche Befunde fanden wir für die Entwicklung von Substanzstörungen. Auch hier zeigen Jugendliche mit einer elterlichen Belastung über die Hochrisikophase hinweg in jedem Lebensalter eine höhere Wahrscheinlichkeit hinsichtlich des Erstauftretens von Missbrauchs- und Abhängigkeitssyndromen. Zusätzlich konnten wir aufzeigen, dass speziell Jugendliche mit zwei betroffenen Eltern früher einen schädlichen Alkoholkonsum entwickeln und auch früher regelmäßig rauchen - somit früher ein problematisches Konsumverhalten diesbezüglich zeigen.

\section{Implikationen der Ergebnisse für die Praxis}

Wie unsere Befunde an einer repräsentativen Stichprobe gezeigt haben, erhöht eine elterliche Alkoholbelastung bereits im Jugendalter das Risiko für die Entwicklung eines problematischen Alkohol- und Drogenkonsums wie auch das Risiko für die Entwicklung von substanzbedingten Missbrauchs- und Abhängigkeitssymptomen. Kinder bzw. Jugendliche mit alkoholbelasteten Eltern sollten deshalb als eine spezifische Risikopopulation betrachtet werden, in welcher bereits in frühen Stadien mittels spezifischer Präventionsund Interventionsmaßnahmen der Suchtentwicklung vorgebeugt werden sollte.

\section{Literatur}

${ }^{1}$ Kessler RC, McGonagle KA, Zhao S et al. Lifetime and 12-month prevalence of DSM-III-R psychiatric disorders in the United States: Results from the National Comorbidity Survey. Arch Gen Psychiatry 1994; 51: 8-19

${ }^{2}$ Nelson CB, Wittchen HU. DSM-IV alcohol disorders in a general population sample of adolescents and young adults. Addiction 1998; 93: 1065-1077

${ }^{3}$ Hawkins JD, Catalano RF, Miller JY. Risk and protective factors for alcohol and other drug problems in adolescence and early adulthood: implications for substance abuse prevention. Psychol Bull 1992; 112: 64-105

${ }^{4}$ Weinberg NZ, Rahdert E, Cilliver JD, Glantz MD. Adolescent substance abuse: A review of the past 10 years. J Am Acad Child Adolesc Psychiatry 1998; 37: 252-261

${ }^{5}$ Sieber M, Angst J. Drogen-, Alkohol- und Tabakkonsum. Ein Beitrag zur Epidemiologie und Ätiologie bei jungen Erwachsenen. Bern: Huber, 1981

${ }^{6}$ Russell M, Cooper ML, Frone MR. The influence of sociodemographic characteristics on familial alcohol problems: data from a community sample. Alcohol Clin Exp Res 1990; 14: 221-226

${ }^{7}$ Sher KJ, Walitzer KS, Wood PK, Brent EE. Characteristics of children of alcoholics: putative risk factors, substance use and abuse, and psychopathology. J Abnorm Psychol 1991; 100: 427-448

${ }^{8}$ Knop J, Goodwin DW, Jensen P et al. A 30-year follow-up study of the sons of alcoholic men. Acta Psychiatr Scand 1993; 370 (Suppl.): 48-53
${ }^{9}$ Mathew RJ, Wilson WH, Blazer DG, George LK. Psychiatric disorders in adult children of alcoholics: data from the Epidemiologic Catchment Area Project. Am J Psychiatry 1993; 150: 793-800

${ }^{10}$ Reich W, Felton E, Frankel O, Shayka JJ. Psychopathology in children of alcoholics. J Am Acad Child Adolesc Psychiatry 1993; 32: 995-1002

${ }^{11}$ Bidaut-Russell M, Bradford SE, Smith EM. Prevalence of mental illness in adult offspring of alcoholic mothers. Drug Alcohol Depend 1994; 35: 81-90

${ }^{12}$ Schuckit MA, Smith TL. An 8-year follow-up of 450 sons of alcoholic and control subjects. Arch Gen Psychiatry 1996; 53: 202-210

${ }^{13}$ Kendler KS, Davis CG, Kessler RC. The familial aggregation of common psychiatric and substance use disorders in the National Comorbidity Survey: a family history study. Br J Psychiatry 1997; 170: 541-548

${ }^{14}$ Dierker LC, Merikangas K, Szatmari P. Influence on parental concordance for psychiatric disorders on psychopathology in offspring. J Am Acad Child Adolesc Psychiatry 1999; 38: 280-288

${ }^{15}$ Hill SY, Yuan H. Familial density of alcoholism and onset of adolescent drinking. J Stud Alcohol 1999; 60: 7-17

${ }^{16}$ Lachner G, Wittchen HU. Familiär übertragene Vulnerabilitätsmerkmale für Alkoholmißbrauch und -abhängigkeit. In: Watzl $\mathrm{H}$, Rockstroh B (Hrsg). Abhängigkeit und Mißbrauch von Alkohol und Drogen. Göttingen: Hogrefe, 1997: 43-90

${ }^{17}$ Zobel M. Kinder aus alkoholbelasteten Familien. Entwicklungsrisiken und -chancen. Göttingen: Hogrefe, 2000

${ }^{18}$ Sher KJ, Gotham HJ, Erickson DJ, Wood PhK. A prospective, high-risk study of the relationship between tobacco dependence and alcohol use disorders. Alcohol Clin Exp Res 1996; 20: 485-492

${ }^{19}$ Windle M. Mate similarity, heavy substance use and family history of problem drinking among young adult women. J Stud Alcohol 1997; 58: 573-580

${ }^{20}$ Kendler KS. Is seeking treatment for depression predicted by a history of depression in relatives? Implications for family studies of affective disorder. Psychol Med 1995; 25: 807-814

${ }^{21}$ Wittchen HU, Perkonigg A, Lachner G, Nelson CB. Early developmental stages of psychopathology study (EDSP): Objectives and design. Eur Addict Res 1998; 4: 18-27

${ }^{22}$ Lieb R, Isensee B, Sydow K von, Wittchen HU. The Early Developmental Stages of Psychopathology Study (EDSP): A methodological update. Eur Addict Res 2000; 6: 170-182

${ }^{23}$ Wittchen HU, Pfister H (Eds). DIA-X-Interviews: Manual für Screening-Verfahren und Interview; PC-Programm zur Durchführung des Interviews (Längs- und Querschnittsuntersuchung); Auswertungsprogramm. Frankfurt: Swets \& Zeitlinger, 1997

${ }^{24}$ World Health Organisation. Composite International Diagnostic Interview (CIDI). Geneva: World Health Organisation, 1990

${ }^{25}$ World Health Organisation. Tenth revision of the international classification of diseases. ICD-10. Chapter V (F): Mental and behavioral disorders. Geneva: World Health Organisation, 1993

${ }^{26}$ American Psychiatric Association. Diagnostic and Statistical Manual of Mental Disorders. Fourth Edition. Washington DC: Author, 1994

${ }^{27}$ Höfler M, Lieb R, Perkonigg A et al. Covariates of cannabis use progression in a representative population sample of adolescents: a prospective examination of vulnerability and risk factors. Addiction 1999; 94: 1679-1694

${ }^{28}$ Reed V, Gander F, Pfister H et al. To what degree the Composite International Diagnostic Interview (CIDI) correctly identifies DSM-IV disorders? Testing validity issues in a clinical sample. Int J Meth Psychiatr Res 1998; 7: 142-155 
${ }^{29}$ Wittchen HU, Lachner G, Wunderlich U, Pfister H. Test-retest reliability of the computerized DSM-IV version of the MunichComposite International Diagnostic Interview (M-CIDI). Social Psychiatry and Psychiatric Epidemiology 1998; 33: 568-578

${ }^{30}$ Lachner G, Wittchen HU, Perkonigg A et al. Structure, content and reliability of the Munich-Composite International Diagnostic Interview (M-CIDI). Substance use sections. Eur Addict Res 1998; 4: 28-41

${ }^{31}$ Holly A, Türk D, Nelson CB et al. Prävalenz von Alkoholkonsum, Alkoholmißbrauch und -abhängigkeit bei Jugendlichen und jungen Erwachsenen. Zeitschr Klin Psychol 1997; 26: 171-178

32 Lieb R, Schuster P, Pfister H et al. Epidemiologie des Konsums, Mißbrauchs und der Abhängigkeit von legalen und illegalen Drogen bei Jugendlichen und jungen Erwachsenen: Die prospektiv-longitudinale Verlaufsstudie EDSP. Sucht 2000; 46: 18-31

33 Perkonigg A, Lieb R, Höfler M et al. Patterns of cannabis use, abuse and dependence over time: incidence, progression and stability in a sample of 1228 adolescents. Addiction 1999; 94: 1663-1678

${ }^{34}$ Sonntag H, Wittchen HU, Höfler M et al. Are social fears and DSM-IV social anxiety disorder associated with smoking and nicotine dependence in adolescents and young adults. Eur Psychiatry 2000; 15: 67-75

${ }^{35}$ Lieb R, Merikangas K, Höfler $\mathrm{M}$ et al. Parental alcohol use disorders and alcohol use and disorders in offspring: a community study. Psychol Med in press

${ }^{36}$ Lachner G, Wittchen HU. Münchener Composite International Diagnostic Interview, M-CIDI (familiengenetische Version), Elternbefragung, Version 2.0. München: Max-Planck-Institut für Psychiatrie, 1997; Eigendruck

${ }^{37}$ Merikangas K, Stevens D, Fenton B et al. Co-morbidity and familial aggregation of alcoholism and anxiety disorder. Psychol Med 1998; 28: 773-788

${ }^{38}$ StataCorp. Stata Statistical Software: Release 7.0. College Station, TX: Stata Corporation. 2001

${ }^{39}$ Fahrmeier L, Tutz G. Multivariate statistical modelling based on generalized linear models. Berlin: Springer, 1994

40 Greenland S. Alternative models for ordinal logistic regression. Statist Medicine 1995; 13: 1665-1677

${ }^{41}$ Andersen PK, Keiding N. Survival Analysis. In: Armitage P, David HA (Hrsg). Advances in Biometry - 50 years of the International Biometric Society. New York: Wiley \& Sons, 1996: 177-200

${ }^{42}$ Kendler KS, Silberg JL, Neale MC et al. The family history method: whose psychiatric history is measured? Am J Psychiatry 1991; 148: 1501-1504

${ }^{43}$ Chapman TF, Mannuzza S, Klein DF, Fyer AJ. Effects of informant mental disorder on psychiatric family history data. Am J Psychiatry 1994; 151: 574-579

${ }^{44}$ Sydow $\mathrm{K}$ von, Lieb R, Pfister $\mathrm{H}$ et al. The natural course of cannabis use, abuse and dependence over four years: A longitudinal community study of adolescents and young adults. Drug Alcohol Dependence in press

${ }^{45}$ Maier W. Mechanismen der familiären Übertragung von Alkoholabhängigkeit und Alkoholabusus. In: Watzl $\mathrm{H}$, Rockstroh $\mathrm{B}$ (Hrsg). Abhängigkeit und Mißbrauch von Alkohol und Drogen. Göttingen: Hogrefe, 1997: 91-110

${ }^{46}$ Lynskey MT, Fergusson DM, Horwood LJ. The effect of parental alcohol problems on rates of adolescent psychiatric disorders. Addiction 1994; 89: 1277-1286

${ }^{47}$ Bierut LJ, Dinwiddie SH, Begleiter $\mathrm{H}$ et al. Familial transmission of substance dependence: alcohol, marijuana, cocaine, and habitual smoking. A report from the collaborative study on the genetics of alcoholism. Arch Gen Psychiatry 1998; 55: 982-988
48 Pollock VE, Schneider LS, Gabrielli WF, Goodwin DW. Sex of parent and offspring in the transmission of alcoholism. A meta-analysis. J Nerv Ment Dis 1987; 175: 668-673

${ }^{49}$ Reich T, Cloninger CR, Vaneerdeweg P, Rice JP, Mullaney J. Secular trends in the familial transmission of alcoholism. Alcoh Clin Exp Res 1988; 12: 458-464

\section{Dr. Roselind Lieb}

Max-Planck-Institut für Psychiatrie Klinische Psychologie und Epidemiologie Kraepelinstraße 2 80804 München

E-mail: lieb@mpipsykl.mpg.de 